Check for updates

Cite this: RSC Adv., 2017, 7, 34307

\section{Wash-free colorimetric homogeneous immunoassay for Zygosaccharomyces rouxii†}

\author{
Hong Guo, Ya Hong Yuan, Chen Niu, Zhouli Wang, Yue Qiu and Tian Li Yue (DD* \\ Convenient, rapid and reliable detection of food spoilage microorganisms has always been critical to food \\ safety. Commonly used methods such as an enzyme-linked immunosorbent assay (ELISA) often rely on \\ tedious wash steps and incubation procedures. A convenient two-step colorimetric homogeneous \\ immunoassay strategy for rapid visual detection of Zygosaccharomyces rouxii, which efficiently \\ simplified the immunoassay process, was developed. Immunogold nanospheres (Ab-PEG-AuNPs) were \\ used as specific colorimetric probes. The proposed method depends on adjusting the interparticle \\ spacing of Ab-PEG-AuNPs on the cell surface of $Z$. rouxii, leading to a visible color change. This simple \\ method exhibits good sensitivity and specificity which depends on the characteristic of Ab against $Z$. \\ rouxii, and the detection results are in great agreement with those of ELISA. Moreover, the method is \\ successfully applied to detect $Z$. rouxii in apple juice.
}

Received 7th March 2017 Accepted 15th June 2017

DOI: 10.1039/c7ra02791e

rsc.li/rsc-advances linked AuNPs-targets-AuNPs lead to an evident optical transition when the inter-spacing of AuNPs-targets-AuNPs is smaller than the diameter of individual AuNPs. However, there is a limit for the detection of larger targets when the interspacing of AuNPs-targets-AuNPs is larger than the diameter of individual AuNPs. Thus, Liu et al. ${ }^{8}$ reported a homogeneous colorimetric assay, wherein the growth of AuNPs could be controlled to decrease the inter-particle spacing of AuNPsprotein-AuNPs. With the addition of Au growth solution, the enlargement of particle size may decrease the inter-particle spacing of the cross-linked AuNPs-protein-AuNPs, thus inducing a distinct optical transition to detect larger proteins. However, this simple method could be limited to detect larger microbes because the inter-spacing of AuNPs-microbe-AuNPs is much larger than that of cross-linked AuNPs-proteinAuNPs.

Thus, we developed the concept of Liu et al. ${ }^{8}$ using specific polyclonal antibodies against microbes to recognize numerous binding sites on the surface of microbes. Therefore, Ab-PEGAuNPs can be focused on the surface of microbes with a closer inter-particle spacing. The microorganism Zygosaccharomyces rouxii has an extreme tolerance to sugar and salt, and it has long been considered a major contributor to food spoilage. ${ }^{\mathbf{1 3 - 1 5}}$ Hence, we herein used polyclonal $Z$. rouxii antibodies, which recognize a large amount of cell surface antigens of $Z$. rouxii, to enrich AuNPs. Upon addition of Au growth solution, Au particles become larger on the yeast cell surface, which may decrease the inter-particle spacing of the AuNPs on the cell surface of $Z$. rouxii, leading to a distinct optical transition (Fig. 1). Finally, we establish a wash-free homogenous colorimetric immunoassay for detection of $Z$. rouxii in apple juice.
College of Food Science and Engineering, Northwest A\&F University, Yangling 712100, China.E-mail: yuetl305@nwsuaf.edu.cn; ytl6503@163.com; Fax: +86-29-87092492; Tel: +86-29-87092492

$\dagger$ Electronic supplementary information (ESI) available. See DOI: 10.1039/c7ra02791e 


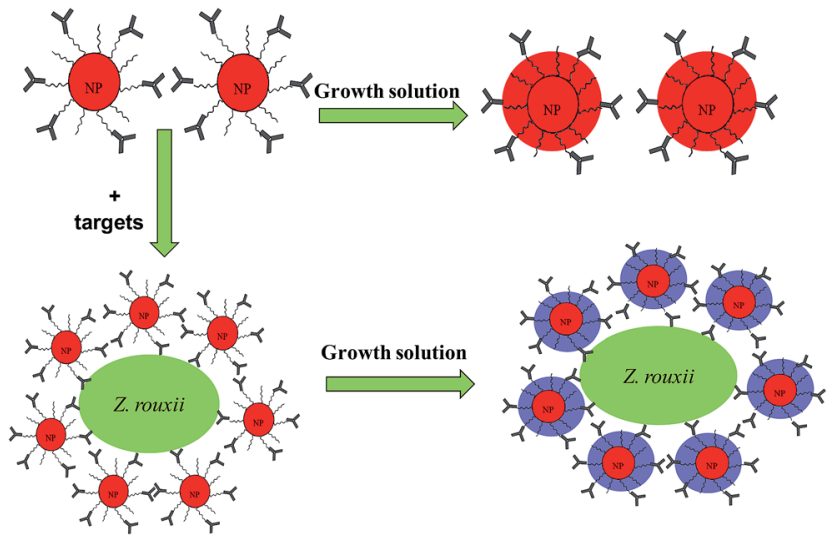

Fig. 1 Schematic of wash-free immunoassay based on the growth of AuNPs.

\section{Materials and methods}

\section{Reagents, materials and instrumentation}

Hydroxylamine $\left(\mathrm{NH}_{2} \mathrm{OH}\right)$, gold(III) chloride trihydrate $\left(\mathrm{HAuCl}_{4}\right.$ $\cdot 3 \mathrm{H}_{2} \mathrm{O}$ ), $\mathrm{N}$-hydroxysuccinimide (NHS), bovine serum albumin (BSA), goat anti-rabbit IgG-horseradish peroxidase (GAR-HRP), 3, $3^{\prime}, 5,5^{\prime}$-tetramethyl benzidine (TMB) (St. Louis, Mo., U.S.A.), and PEG 2000 derivative $\left(\mathrm{H}_{2} \mathrm{~N}-\mathrm{PEG}-\mathrm{COOH}\right)$ were purchased from Sigma. Trisodium citrate, thioctic acid, PBS ( $\mathrm{pH} 7.4$ ), and 4-dimethylaminopyridine (DMAP) of high purity were purchased locally. ELISA was carried out in 96-well polystyrene microplates (Nunc, Roskilde, Denmark). The UV-vis spectra were recorded with U-2500 spectrophotometer (Shimadzu). The absorbance of AuNP solutions in 96-well microplates was observed at $528 \mathrm{~nm}$. TEM images were obtained using a JEOL1230 TEM at an accelerating voltage of $100 \mathrm{kV}$.

\section{Preparation of antigen}

The strain $Z$. rouxii B-WHX-12-54 used in this study was previously isolated from apple juice concentrate ${ }^{\mathbf{1 6}}$ and was cultured in YPD medium (1\% yeast extract, $2 \%$ bacto-peptone and $2 \%$ glucose) and $60 \%$ YPD medium (1\% yeast extract, $2 \%$ bacto-peptone and $60 \%$ glucose) ${ }^{17}$ at $28{ }^{\circ} \mathrm{C}$ and $120 \mathrm{rpm}$ for $48 \mathrm{~h}$. Yeast cells grown in $60 \%$ YPD medium (h- $Z$. rouxii) were harvested by centrifugation and washed three times with sterile water, and the final cell concentration was approxi-

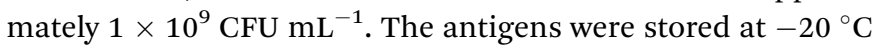
until use. ${ }^{18}$

\section{Preparation of immunogold colorimetric probe}

Antibody production. Immunization was performed according to reported methods. ${ }^{19}$ Briefly, two New Zealand White male rabbits were inoculated by ear-vein intravenous injection with $1 \mathrm{~mL}$ antigen (Z. rouxii cells) at $10^{9} \mathrm{CFU} \mathrm{mL}^{-1}$, followed by injection of $2 \mathrm{~mL}$ antigen on six subsequent occasions at four-day intervals. After the final immunization, the serum of the inoculated rabbits was collected and pretreated by a saturated ammonium sulfate (SAS) precipitation method. The polyclonal antibody (Ab) was purified and then divided into aliquots and finally stored at $-20{ }^{\circ} \mathrm{C} .{ }^{18}$

Treated polyclonal antibody (tAb) was prepared by the methods described by Casanova ${ }^{20}$ with minor modifications. Briefly, the Ab was adsorbed three times with $6 \mathrm{mg}$ dried heatkilled $Z$. rouxii B-WHX-12-54 grown in YPD medium (1- $Z$. rouxii) and stored at $-20{ }^{\circ} \mathrm{C}$ until used. The animal protocol was approved by the laboratory animal management committee of Shanghai. All of the experimental procedures followed the Guide for the Care and Use of Laboratory Animals: eighth edition, ISBN-10: 0-309-15396-4. The housing facility was a barrier housing facility, and it met the criteria laid out by the National Standard of China, Laboratory Animal-Requirements of Environment and Housing Facilities (GB 14925-2010/XG12011). All efforts were made to minimize animal suffering. Informed consent: not applicable.

Synthesis of gold nanoparticles. Citrate coated gold nanoparticles were synthesized using the Turkevich method ${ }^{21}$ as reported in a previous study. Briefly, to a boiling solution of 100 $\mathrm{mL} \mathrm{HAuCl}_{4}(0.01 \% \mathrm{w} / \mathrm{v}), 5 \mathrm{~mL} \mathrm{1 \%}$ trisodium citrate was added. After boiling for $10 \mathrm{~min}$, the solution was cooled to the room temperature for use.

Synthesis of PEG linker. The PEG linker was synthesized according to the method recently described by Liu. ${ }^{8}$ Briefly, $0.67 \mathrm{~g}$ thioctic acid was added into $10 \mathrm{~mL}$ anhydrous dichloromethane containing $0.45 \mathrm{~g}$ NHS while stirring. Then, EDC-HCl $(0.69 \mathrm{~g})$ and catalyst DMAP $(\sim 0.2 \mathrm{~g})$ were added. The mixed solution was cooled for $1 \mathrm{~h}$ and allowed to react overnight at room temperature. The resulting solution was diluted with dichloromethane $(15 \mathrm{~mL})$ and washed with saturated brine (25 $\mathrm{mL}$ ) several times. Then, the mixture was dried using $\mathrm{Na}_{2} \mathrm{SO}_{4}$ to produce an NHS-activated ester. The activated ester (91 $\mathrm{mg}$ ) was added to a solution of $\mathrm{H}_{2} \mathrm{~N}-\mathrm{PEG}-\mathrm{COOH}(0.5 \mathrm{~g})$ in dichloromethane $(15 \mathrm{~mL})$ and stirred, and the solution was left to react overnight. The obtained solution was concentrated to $5 \mathrm{~mL}$ and then cold diethyl ether $(25 \mathrm{~mL})$ was added dropwise to obtain a precipitate. Finally, we obtained a PEG linker in which one side of the heterobifunctional $\mathrm{H}_{2} \mathrm{~N}-\mathrm{PEG}-\mathrm{COOH}$ reacted with thioctic acid and the other side was carboxylic acid activated by $\mathrm{N}$-hydroxysuccinimide (NHS).

Synthesis of Ab-PEG-AuNP colorimetric probe. $10 \mathrm{~mL}$ asprepared AuNP was incubated with $0.003 \mathrm{~g}$ PEG linker at room temperature for $2 \mathrm{~h}$. The mixture was purified by centrifugation (1100 rpm, $20 \mathrm{~min}$ ) and resuspended twice in PBS. Then, $4 \mathrm{~mL}$ of the purified solution was incubated with $16 \mu \mathrm{L} \mathrm{h}$ Z. rouxii polyclonal antibody $\left(6.3 \mathrm{mg} \mathrm{mL}^{-1}\right)$ at $4{ }^{\circ} \mathrm{C}$ overnight. In order to hydrolyse the unspecific site of NHS, $0.2 \mathrm{~mL}$ PBS (pH 8.5) was added. Then, Ab-PEG-AuNPs were purified by centrifugation and resuspended in ultra-pure water to form a colorimetric probe stock solution.

\section{Procedure of wash-free colorimetric immunoassay for $Z$. rouxii detection in PBS buffer}

The wash-free immunoassay was used to detect $Z$. rouxii by two steps without washing. In the first step, the as-prepared AbPEG-AuNP colorimetric probe (diluted to $1 \mathrm{nM}$ ) was incubated 
with $Z$. rouxii at $37{ }^{\circ} \mathrm{C}$ for $30 \mathrm{~min}$. Second, Au growth solutions $10 \mu \mathrm{L} \mathrm{NH}{ }_{2} \mathrm{OH}\left(400 \mathrm{mM}\right.$; pH, 5) and $8 \mu \mathrm{L} \mathrm{HAuCl}_{4}(0.1 \mathrm{w} / \mathrm{v} \%)$ were added to the analysis system. The color of the Ab-PEG-AuNP solution changed from light pink to red or blue. AuNP growth is a dynamic process and leads to increasing color intensity with time. As AuNPs usually precipitate absolutely after 120 s, visible color changes were recorded within 25-120 s. To ensure consistency of results, all the wash-free immunoassay photos were taken within $25-120 \mathrm{~s}$.

\section{ELISA analysis}

The procedure used for ELISA analysis was according to that provided by Wang et al. ${ }^{22}$ with minor modifications. In our process, 96-well microtiter plates were coated with $100 \mu \mathrm{L}$ sample for $1 \mathrm{~h}$ at $37{ }^{\circ} \mathrm{C}$. After incubation, the plates were washed three times with $200 \mu \mathrm{L} 10 \mathrm{mM}$ PBS (pH 7.4) with $0.05 \%$ Tween-20. Then, the plates were incubated with blocking buffer, $5 \%$ milk powder in $10 \mathrm{mM}$ PBS at $37{ }^{\circ} \mathrm{C}$, for $2 \mathrm{~h}(200$ $\mu \mathrm{L}$ per well). After three washes, the wells were blotted on adsorbent paper. Then, $100 \mu \mathrm{L}$ of diluted antibody $(1: 4000$ in PBS) was added. Following additional incubation for $1 \mathrm{~h}$ at $37{ }^{\circ} \mathrm{C}$, the plate was washed again. GAR-HRP was then added (1 : 2000 in PBS; $100 \mu \mathrm{L}$ per well) and the plate was incubated at $37{ }^{\circ} \mathrm{C}$ for $1 \mathrm{~h}$. After three washes, substrate solution A $\left(3,3^{\prime}, 5,5^{\prime}\right.$-tetramethylbenzidine (TMB)) and substrate solution $\mathrm{B}\left(0.03 \% \mathrm{H}_{2} \mathrm{O}_{2}\right)$ were added to each well $(100 \mu \mathrm{L})$ to allow color development for $15 \mathrm{~min}$ under dark conditions at $37{ }^{\circ} \mathrm{C}$. The reaction was stopped by adding $50 \mu \mathrm{L}$ of sulfuric acid $(2 \mathrm{M})$. The optical density of each well was measured at $450 \mathrm{~nm}$ by a microtiter plate reader (Bio-Rad Laboratories. Inc. Hercules. CA. USA).

\section{Immunofluorescence studies}

The method of immunolabeling of $Z$. rouxii grown under different sugar stresses $(10 \%, 20 \%, 30 \%, 40 \%$ and $60 \%$ YPD media) was according to that reported by Beaussart et al. ${ }^{23}$ Images were obtained using an Andor CSU-W confocal microscopy system. A $100 \times$ oil objective with a DU-888U3-CSO was used for all images that were captured with Andor iQ3 live cell imaging software.

\section{Apple juice samples}

The $Z$. rouxii B-WHX-12-54 was cultured in YPD at $28^{\circ} \mathrm{C}$ for $48 \mathrm{~h}$ to produce working stock solution and stored at $4{ }^{\circ} \mathrm{C}$ for routine use. Moreover, $100 \mu \mathrm{L}$ of cells from the working stock solution were incubated into $12^{\circ}$ Brix, $25^{\circ}$ Brix, $34^{\circ}$ Brix, $42^{\circ}$ Brix, and $59^{\circ}$ Brix apple juice and allowed to grow. The apple juice samples were then detected using the wash-free immunoassay based on AuNPs.

\section{Live subject statement}

The animal protocol was approved by the laboratory animal management committee of Shanghai. All of the experimental procedures followed the Guide for the Care and Use of Laboratory Animals: eighth edition, ISBN-10: 0-309-15396-4.

\section{Results and discussion}

\section{Principle of wash-free immunoassay}

We developed the concept of immunoassay protein, as reported by Liu et $a .^{8}$ to qualitatively detect larger microbes. The principle of the established immunoassay method in this study to detect microbes is summarized in Fig. 1. To investigate the feasibility of the wash-free colorimetric immunoassay, we prepared AuNPs and a PEG linker. One of the side of PEG linker containing a dithiol group was functionalized onto AuNPs surface via Au-S to form PEG-AuNPs. AuNPs and Ab-PEGAuNPs can be used as seeds to grow larger AuNPs by addition of $\mathrm{Au}$ growth solutions $\left(\mathrm{NH}_{4} \mathrm{OH}\right.$ and $\left.\mathrm{HAuCL}_{4}\right) .{ }^{8}$ To confirm this, the growth of AuNPs and PEG-AuNPs was first investigated. In this study, AuNPs and PEG-AuNPs were diluted 5 fold in Milli Q water (about $1 \mathrm{nM}$ ) and added to $\mathrm{Au}$ growth solution $\left(\mathrm{NH}_{4} \mathrm{OH}\right.$ $(10 \mu \mathrm{L})$ and $\left.\mathrm{HAuCL}_{4}(8 \mu \mathrm{L}, 0.01 \% \mathrm{w} / \mathrm{v})\right)$. As indicated in Fig. S2, $\dagger$ AuNPs contain several cross-linked aggregates, while PEGAuNPs show mostly individual NPs, indicating the role of the PEG linker in dispersion formation. After introduction of $\mathrm{Au}$ growth solutions, the resulting solutions of AuNPs became blue, and the color of PEG-AuNP solutions turned red. Corresponding TEM images of AuNPs showed undesirable aggregates (Fig. 2c), while PEG-AuNPs exhibited good dispersion (Fig. 2d). The synthetic AuNPs in PEG-AuNPs with a diameter of about $13 \mathrm{~nm}$ at $200000 \times$ magnification display spherical morphology and grow to about $30 \mathrm{~nm}$ after addition of Au growth solutions. The highly stable dispersions of PEG-AuNPs were consistent with those observed in a previous study, which showed that PEGylated gold nanoparticle bioconjugates form highly stable dispersions. ${ }^{24}$ Therefore, PEG-AuNPs were selected to prepare the colorimetric probe for further study.

We then used the PEG-AuNPs to conjugate Ab to establish the AuNP-based immunoassay. Ab is usually stored at $-20{ }^{\circ} \mathrm{C}$ to maintain its good biological activity. As the formation of Abconjugated PEG-AuNPs requires only two simple steps, we evaluated the stability of PEG-AuNPs. After storage of the PEGAuNPs in a refrigerator for several months at $4{ }^{\circ} \mathrm{C}$, we were able to obtain the same results as shown in Fig. $\mathrm{S} 2, \uparrow$ which indicate high stability and shelf life of the conjugates. PEG-AuNPs were stored at $4{ }^{\circ} \mathrm{C}$ for routine use. In this study, polyclonal antibodies against $Z$. rouxii $(\mathrm{Ab})$ were obtained. $\mathrm{Ab}$ was then conjugated with the PEG-AuNPs by means of amide bonds to form Ab-PEG-AuNPs. Fig. 2 shows the step-by-step modification of AuNPs. As shown in Fig. S1, $\dagger$ after conjugation with Ab, the absorbance peak of AuNPs red-shifts from $518 \mathrm{~nm}$ to $522 \mathrm{~nm}$. The difference of the resonance peak is due to the local increase in the reflective index caused by the addition of antibody layers. ${ }^{25}$ This information indicates the successful preparation of Ab-PEG-AuNPs.

Herein, specific polyclonal antibodies were used to recognize the cell surface antigens of target microbes, with AuNPs act as the signal indicator. PEG is used to stabilize AuNPs to prevent their aggregation. Thus, Ab specifically recognizes $Z$. rouxii and subsequently anchors Ab-PEG-AuNPs complexes on it (Fig. 1). The Ab-PEG-AuNPs will disperse in the absence of the target 
a)<smiles>CC(C)(C)OCCC(C)(C)ON1C(=O)CCC1=O</smiles>

PEG-linker

b)

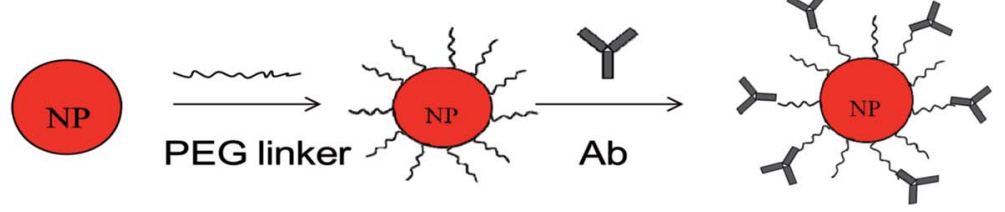

Fig. 2 (a) Structure of PEG linker; (b) step-by-step assembly of Ab-PEG-AuNPs.

strain and will focus on the cell surface of $Z$. rouxii. At this time, enrichment of AuNPs on the surface of $Z$. rouxii does not cause a color change because the inter-particle distance of AuNPs is still larger than the diameter of individual AuNPs. ${ }^{8}$ After the introduction of growth solutions to the analysis system, the diameter of individual AuNPs increased to around $30 \mathrm{~nm}$ with dispersion, which was similar to the result shown in Fig. $2 d$ in the absence of a target, resulting in a deep-red solution. In contrast, when the $Z$. rouxii B-WHX-12-54 is present in the AbPEG-AuNP solution, the forming of large-sized AuNPs may decrease the inter-particle spacing of AuNPs on the cell surface of $Z$. rouxii with large-scale aggregation, resulting in a blue color.

\section{Wash-free colorimetric immunoassay for Z. rouxii detection in PBS}

We now investigate the detection of $Z$. rouxii $\mathrm{B}-\mathrm{WHX}-54$ in $\mathrm{PBS}$ (pH 7.4). First, blank PBS $(3 \mu \mathrm{L})$ and $10^{5} \mathrm{CFU} \mathrm{mL}^{-1} Z$. rouxii BWHX-12-54 (3 $\mu \mathrm{L})$ were incubated with Ab-PEG-AuNP solution $(600 \mu \mathrm{L}, 1 \mathrm{nM})$ at $37{ }^{\circ} \mathrm{C}$ for $30 \mathrm{~min}$. The color and maximum absorption peak of the analysis system were not significantly changed (Fig. 3a and b). It has been reported that the mixing of small biological molecular targets with Ab-AuNPs would cause cross-linking of Ab-AuNP probes to form aggregates, leading to a red-shift. ${ }^{11,12,23}$ The different results between the larger microorganisms and small analyte is due to the inter-particle

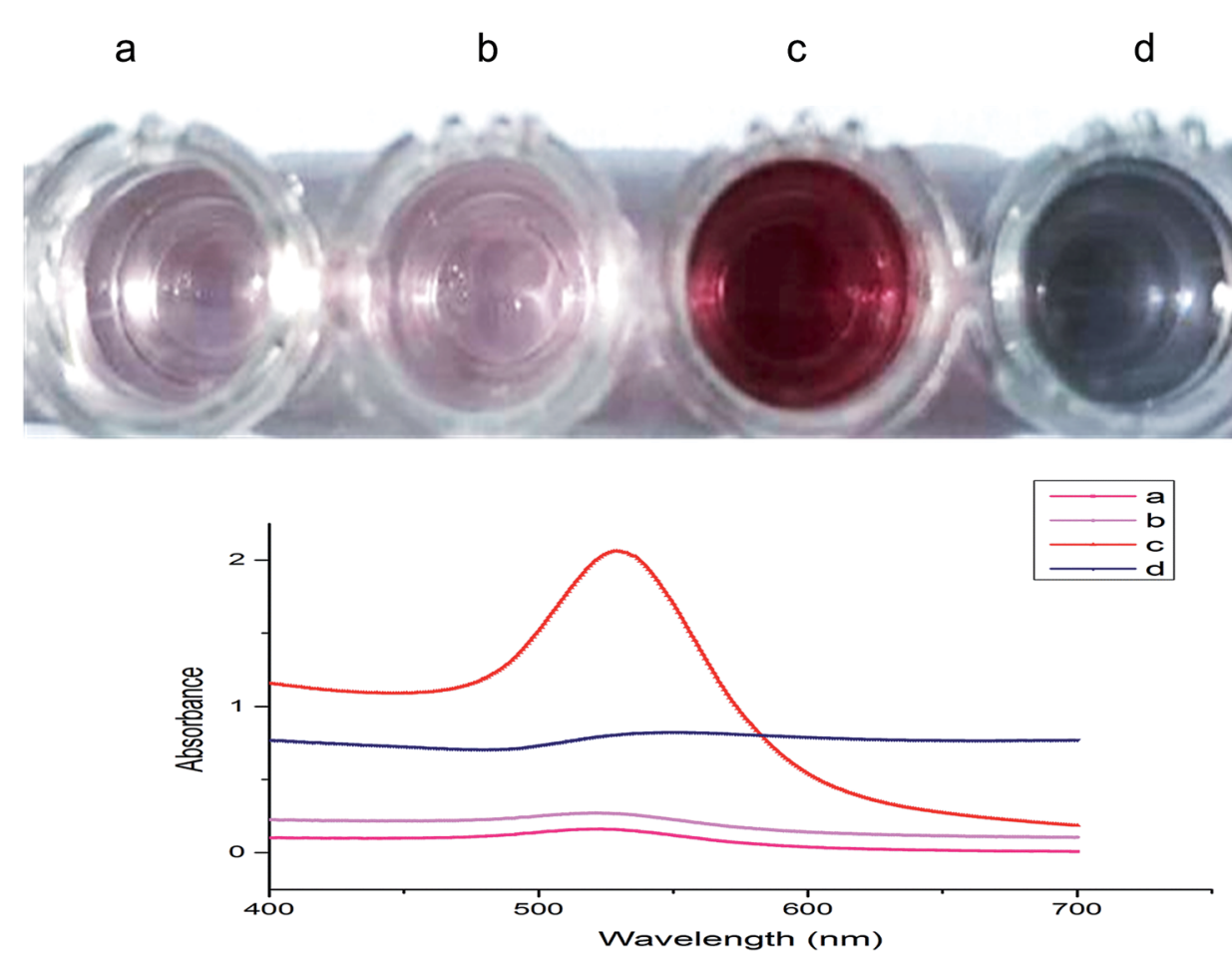

Fig. 3 UV-vis spectra and corresponding photographs of analytical solutions. (a) Ab-PEG-AuNP; (b) (a) incubated with Z. rouxii; (c) (a) +Au growth solution; (d) (b) +Au growth solution. 
spacing of AuNPs. After addition of Au growth solutions, $\mathrm{Ab}-$ PEG-AuNPs grow into a larger-sized AuNPs with dispersions in the absence of targets, resulting in a deep-red solution (Fig. 3c). The corresponding absorbance at $528 \mathrm{~nm}$ increased from 0.161 to 2.063. This is because the free dispersion of Ab-PEG-AuNPs does not decrease the inter-particle spacing of AuNPs. Conversely, the introduction of growth solutions to the $\mathrm{Ab}-$ PEG-AuNPs- $Z$. rouxii complex caused AuNP aggregation, resulting in a blue solution (Fig. 3d). We speculated that the growth of AuNPs decreases the interparticle spacing of the AbPEG-AuNPs on the surface of the $Z$. rouxii, which was verified by a distinct wavelength red-shift of SPR. As a result, the absorption band shifted from $528 \mathrm{~nm}$ to $554 \mathrm{~nm}$ with increased SPR intensity. Undoubtedly, this method can detect spoilage yeast by mixing sample and colorimetric probe followed by introduction of Au growth solutions, without the involvement of any enzymes and tedious wash steps.

\section{Sensitivity, specificity and reliability of wash-free immunoassay}

The sensitivity of the wash-free colorimetric immunoassay for $Z$. rouxii detection was evaluated in PBS (pH 7.4). The Ab-PEGAuNPs (diluted to $1 \mathrm{nM}$ ) were incubated with various amounts of $\mathrm{h}-Z$. rouxii $\mathrm{B}-\mathrm{WHX}-12-54$ and $\mathrm{l}-\mathrm{Z}$. rouxii $\mathrm{B}-\mathrm{WHX}-12-54$ cells with final concentrations of $10^{1}, 10^{2}, 10^{3}$, and $10^{4} \mathrm{CFU} \mathrm{mL} \mathrm{m}^{-1}$ in PBS at $37^{\circ} \mathrm{C}$ for $30 \mathrm{~min}$. On addition of Au growth solutions, as indicated in Fig. 4a, the color changed to blue when the analytical system contained $10^{3} \mathrm{CFU} \mathrm{mL}^{-1}$ of h-Z. rouxii B-WHX12-54. The color change was measured by absorbance at $528 \mathrm{~nm}$. By plotting $-\Delta A_{528}\left(-\Delta A_{528}=A-A_{0}\right.$, where $A$ is the absorbance of the Ab-PEG-AuNP solution at $528 \mathrm{~nm}$ after adding targets and Au growth solutions and $A_{0}$ is that of the blank sample) versus various concentrations of $Z$. rouxii, Fig. $4 \mathrm{~b}$ was achieved. The results indicated that the colorimetric probe is sensitive to $Z$. rouxii B-WHX-54. However, the polyclonal h- $Z$.

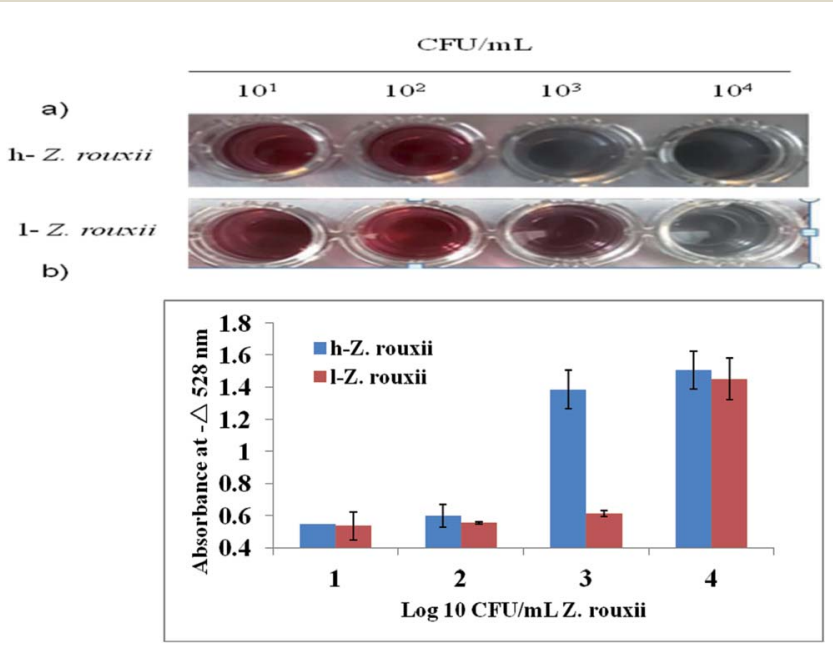

Fig. 4 Photographs (a) and typical absorption spectral response (b) of wash-free immunoassay at various concentrations of $Z$. rouxii. Error bars denote the standard deviation of the absorbance values from the three replicate assays. rouxii antibody showed a different affinity for sensing $Z$. rouxii, being able to sense $10^{3} \mathrm{CFU} \mathrm{mL} \mathrm{m}^{-1}$ of $\mathrm{h}-Z$. rouxii and $10^{4} \mathrm{CFU}$ $\mathrm{mL}^{-1}$ of $1-Z$. rouxii. One of the reasons could be that the affinity of the antibody is related to the analyte structure. ${ }^{26,27}$ Since stress conditions can affect the structure of microorganisms, ${ }^{28}$ $\mathrm{Ab}$ could have different affinity to $Z$. rouxii grown under different stresses. Another reason could be that the cell proteins usually change when subjected to sugar stress. Kim et al..$^{29}$ have suggested that Candida magnoliae grown in $300 \mathrm{~g} \mathrm{~L}^{-1}$ glucose medium will cause protein changes. Recently, we also demonstrated that $Z$. rouxii grown in a high sugar content medium (600 $\mathrm{g} \mathrm{L}^{-1}$ glucose) will vastly reprogram proteins. ${ }^{17}$

Subsequently, the specificity of the wash-free immunoassay was tested via incubation of analysis solutions with $\mathrm{h}-Z$. rouxii, $\mathrm{l}$ Z. rouxii, Zygosaccharomyces bailii, Debaryomyces hansenii, Hanseniaspora uvarum, and Saccharomyces cerevisiae. Except h$Z$. rouxii, all test strains were grown in YPD media and washed with PBS. Upon addition of Au growth solutions, only h-Z. rouxii and $1-Z$. rouxii displayed a blue colour (Fig. 5a), and the adsorption peaks shifted significantly (Fig. 5b), suggesting the high selective performance of the probe.

Before applying the AuNP-based method to detect different concentrations of apple juice (different sugar content), we immunostained $Z$. rouxii B-WHX-54 under different sugar stress conditions (Fig. 6). Although the morphology of $Z$. rouxii BWHX-54 significantly changed, homogeneous fluorescence was found on the cell surface of $Z$. rouxii B-WHX-54, which suggested that $\mathrm{Ab}$ could identify several binding sites on the surface of $Z$. rouxii B-WHX- 54 . These results confirm the reliability of the principle of the wash-free colorimetric immunoassay.

\section{Detection of $Z$. rouxii in apple juice}

With these promising results, this method was applied to detect Z. rouxii B-WHX-12-54 in apple juice. $Z$. rouxii B-WHX-12-54
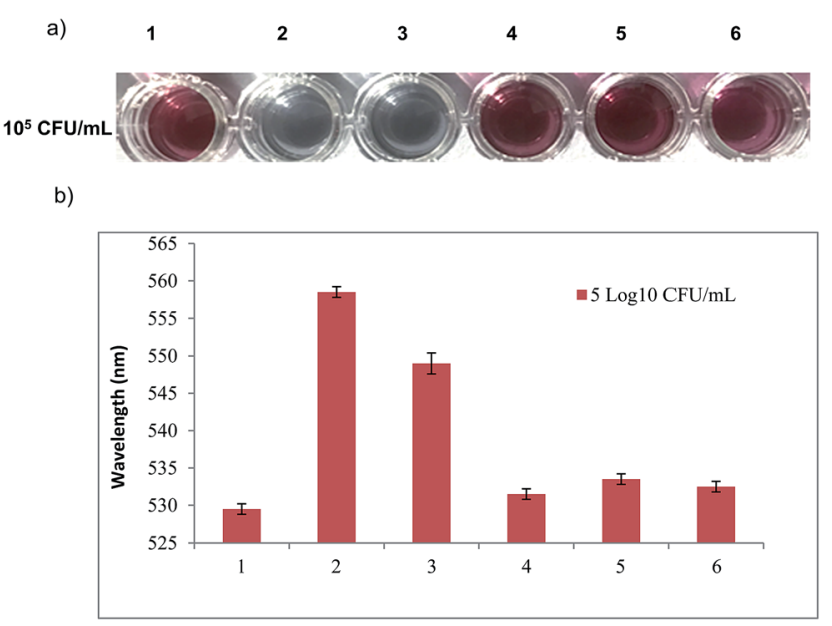

Fig. 5 Photographs (a) and typical absorption spectral response (b) of wash free immunoassay various tested strains. 1 blank, $2 \mathrm{~h}-Z$. rouxii, 3 I-Z. rouxii, 4 Z. bailli, 5 D. hansenii, 6 S. cereviae. Error bars in (b) denote the standard deviation of the wavelength. 

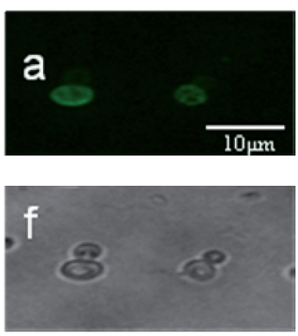
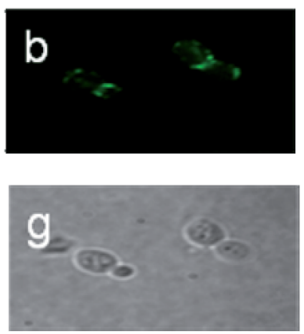
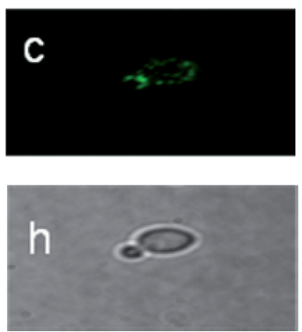
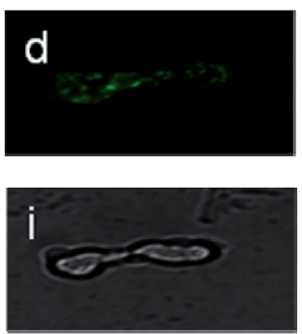
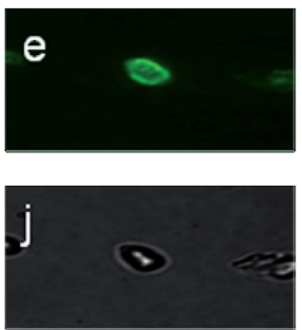

Fig. 6 Fluorescence imaging of Z. rouxii B-WHX-12-54 grown at different sugar concentrations. Fluorescence (a-e) and phase-contrast (f-j) images of Z. rouxii BW-WHX-12-54 grown in 10\%, 20\%, 30\%, 40\% and 60\% YPD media, stained with anti-Z. rouxii pAb, followed by FITCconjugated secondary antibodies.

grown at different concentrations of apple juice $\left(12^{\circ} \mathrm{Brix}, 25^{\circ}\right.$ Brix, $34^{\circ}$ Brix, $42^{\circ}$ Brix, and $59^{\circ}$ Brix) was incubated with $\mathrm{Ab}-$ PEG-AuNP solutions with final yeast concentrations of $0,10^{3}$, $10^{4}$, and $10^{5} \mathrm{CFU} \mathrm{mL}{ }^{-1}$. As indicated in Fig. S3, $\uparrow$ the wash-free method can be used for detecting $Z$. rouxii in $12^{\circ}$ Brix apple juice, while the detection was seriously disturbed in $25-59^{\circ}$ Brix apple juice. The reason could be that the polyclonal antibodies can cross-react with antigenic matrix in apple juice.

To reduce the cross-reactivity of the antibody, Casanova et al. ${ }^{20}$ demonstrated that C. albicans polyclonal antibodies were adsorbed with blastoconidia to avoid cross-reaction of the latter. Therefore, we try to obtain the treatment antibody (tAb) using $\mathrm{l}-Z$. rouxii to adsorb $\mathrm{Ab}$ and evaluate whether it can avoid

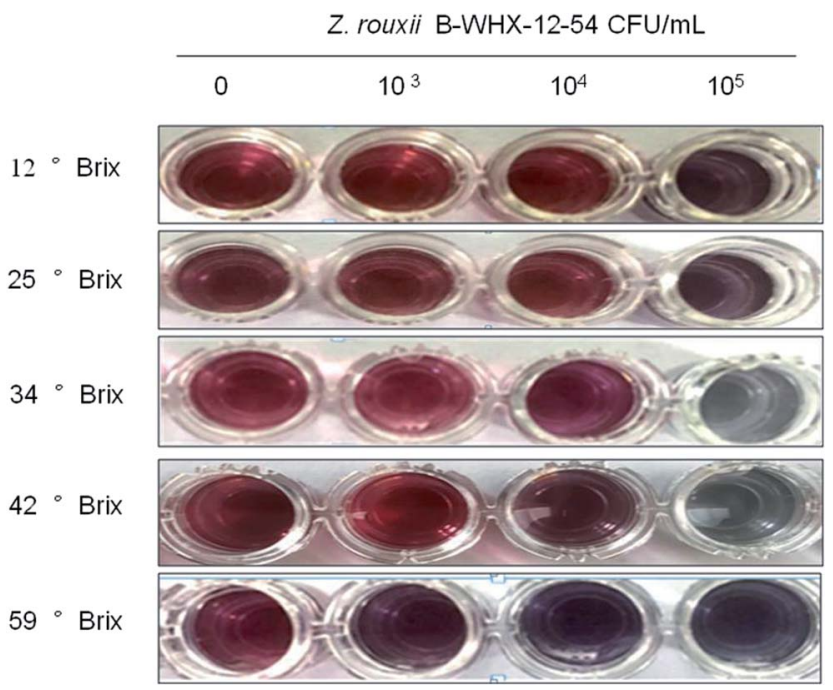

Fig. 7 The naked eye observations of wash-free immunoassay (tAbPEG-AuNPs) for $Z$. rouxii at different concentrations of apple juice. cross-reaction. Then, the obtained tAb is used for the synthesis of tAb-PEG-AuNP probes, as described in the materials and methods. We then use the tAb-PEG-AuNP solution to detect $Z$. rouxii B-WHX-12-54 at different concentrations of apple juice. The results in Fig. 7 showed that tAb-PEG-AuNPs can be used for detection of $12-42^{\circ}$ Brix apple juice. Detection limit was determined to be $10^{4} \mathrm{CFU} \mathrm{mL}^{-1}$ of $Z$. rouxii in $42^{\circ}$ Brix apple juice and $10^{5} \mathrm{CFU} \mathrm{mL}^{-1}$ of $Z$. rouxii in apple juice with concentration ranging from 12 to $34^{\circ}$ Brix, which led to visible color changes. The reason may be that the tAb avoids the crossreaction of food matrix in apple juice because some cell surface antigenic compositions of $1-Z$. rouxii may be similar to sugar compositions in apple juice. Therefore, these polyclonal antibodies, which cross-react with apple juice, are removed by adsorption of $1-Z$. rouxii. These results suggest that the adsorbed antibodies facilitate the wash-free immunoassay method to detect $Z$. rouxii in a broad range of apple juice concentrations. The tAb used in the ELISA test, shown in Table 1, agrees with that used in the PEG-AuNP-based method. For $59^{\circ}$ Brix apple juice, ELISA test results showed negative results irrespective of the presence of $Z$. rouxii B-WHX-54. The reason may be that $Z$.

Table 1 Samples measured by conventional indirect ELISA using tAb

\begin{tabular}{lllll}
\hline & \multicolumn{4}{l}{ Z. rouxii B-WHX-54 } \\
\cline { 2 - 5 } ELISA & 0 & $10^{3}$ & $10^{4}$ & $10^{5}$ \\
\hline $12^{\circ}$ Brix & $-(0.1)^{a}$ & $-(0.10)$ & $-(0.11)$ & $-(0.20)$ \\
$25^{\circ}$ Brix & $-(0.21)$ & $-(0.21)$ & $-(0.24)$ & $-(0.24)$ \\
$34^{\circ}$ Brix & $-(0.218)$ & $-(0.244)$ & $-(0.24)$ & $+(0.34)^{b}$ \\
$42^{\circ}$ Brix & $-(0.22)$ & $-(0.23)$ & $-(0.24)$ & $+(0.36)$ \\
$59^{\circ}$ Brix & $-(0.19)$ & $-(0.22)$ & $-(0.21)$ & $-(0.22)$
\end{tabular}

${ }^{a}$ Negative. ${ }^{b}$ Positive. 
Table 2 Comparison between our method and conventional ELISA method

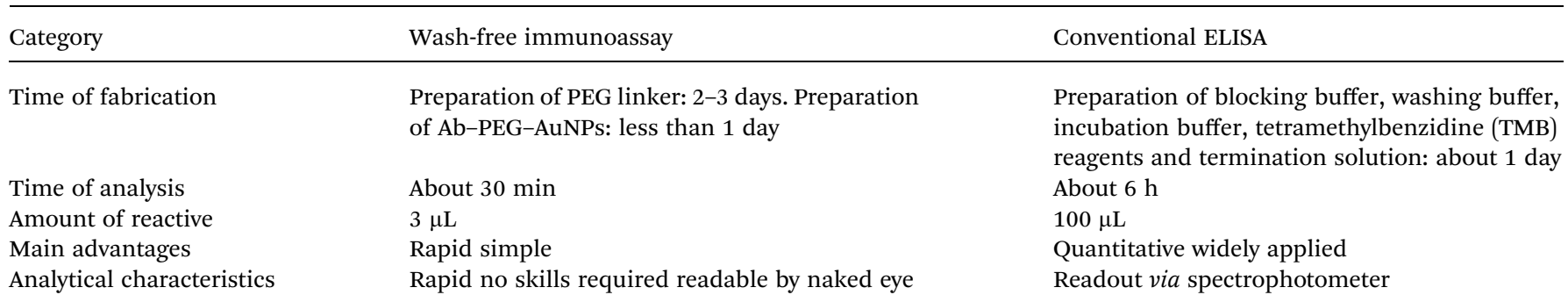

rouxii will float on the surface of $59^{\circ}$ Brix apple juice because of the high viscosity of concentrated juice. Therefore, Z. rouxii BWHX-54 cannot attach to the bottom of 96-well polystyrene microplates (Fig. S4 $\dagger$ ), exhibiting negative results. However, tAb-PEG-AuNPs will inevitably cross-react when the molecular concentration is sufficiently large or the viscosity affects the Au growth in the analysis system, so that the wash-free immunoassay shows a positive result regardless of the presence of targets. Unambiguously, tAb-PEG-AuNPs might hold promising advantages for the determination of $Z$. rouxii in apple juice (Fig. S4 $\dagger$ ). In addition, the detailed comparison of the AuNPbased method and ELISA method shown in Table 2 help developers make proper choices.

Based on these results, we indicate that antibodies are the key factor in the wash-free immunoassay when this method is applied for the detection of other food spoilage microbes. Compared with other methods for detecting spoilage microbes, ELISA $^{6}$ demanded tedious wash steps, E-nose ${ }^{13}$ and RT-PCR ${ }^{30}$ required high personal skills, and the plating ${ }^{31}$ method was time consuming; conversely, the established method in our study was simple, sensitive and fast. Thus, it has great potential for detecting other food spoilage microorganisms.

\section{Conclusion}

The wash-free immunoassay strategy based on AuNP-growth can be applied in rapid detection of $Z$. rouxii in apple juice. The polyclonal antibodies were assembled on AuNPs to recognize the cell surface antigens of $Z$. rouxii. Upon addition of Au growth solutions, the inter-particle spacing of Ab-PEG-AuNP on the cell surface of $Z$. rouxii was reduced depending on the Au growth, leading to an optical transition. Therefore, the color change of the analysis system was observed by simply mixing real samples and Ab-PEG-AuNP solutions, followed by addition of $\mathrm{Au}$ growth solutions. This method does not need tedious wash steps, the involvement of any enzyme and the separation of targets. The concept used in this study can be expanded to food spoilage microbe detection and only requires to change the $\mathrm{Ab}$ in the Ab-PEG-AuNP probe.

\section{Acknowledgements}

This study was financially supported by the Shaanxi Special Project of China (2016KTCQ03-12), the National Natural Science Foundation of China (31371814, 31671866), and the Scientific and Technology Cooperation Project in Hongkong, Macao and Taiwan of China (2015DFT30130).

\section{References}

1 B. K. Kumar, P. Raghunath, D. Devegowda, V. K. Deekshit, M. N. Venugopal, I. Karunasagar and I. Karunasagar, Int. J. Food Microbiol., 2011, 145, 244-249.

2 C. Pastells, G. Acosta, N. Pascual, F. Albericio, M. Royo and M. P. Marco, Anal. Chim. Acta, 2015, 889, 203-211.

3 Z. Wang, R. Cai, Y. Yuan, C. Niu, Z. Hu and T. Yue, Int. J. Food Microbiol., 2014, 175, 30-35.

4 W. Xu, J. Wang and Q. Li, FEMS Yeast Res., 2014, 14, 12731285.

5 J. Li, K. Xia and C. Yu, Food Control, 2013, 30, 251-254.

6 J. Li, R. Huang, K. Xia and L. Liu, Food Control, 2014, 40, 172176.

7 H. Song, H. Xue and Y. Han, Food Control, 2011, 22, 883-887.

8 H. Liu, P. Rong, H. Jia, J. Yang, B. Dong, Q. Dong, C. Yang, P. Hu, W. Wang and H. Liu, Theranostics, 2016, 6, 54-64.

9 X. M. Nie, R. Huang, C. X. Dong, L. J. Tang, R. Gui and J. H. Jiang, Biosens. Bioelectron., 2014, 58, 314-319.

10 X. Bai, C. Shao, X. Han, Y. Li, Y. Guan and Z. Deng, Biosens. Bioelectron., 2010, 25, 1984-1988.

11 J.-S. Lee, P. A. Ulmann, M. S. Han and C. A. Mirkin, Nano Lett., 2008, 8, 529-533.

12 J. Liu and Y. Lu, J. Am. Chem. Soc., 2003, 125, 6642-6643.

13 H. Wang, Z. Hu, F. Long, C. Guo, Y. Yuan and T. Yue, Int. J. Food Microbiol., 2016, 217, 68-78.

14 T. C. Dakal, L. Solieri and P. Giudici, Int. J. Food Microbiol., 2014, 185, 140-157.

15 J. Watanabe, K. Uehara and Y. Mogi, Genetics, 2013, 195, 393-405.

16 H. Wang, Z. Hu, F. Long, C. Niu, Y. Yuan and T. Yue, J. Food Sci., 2015, 80, M1850-M1860.

17 H. Guo, C. Niu, B. Liu, J. Wei, H. Wang, Y. Yuan and T. Yue, Int. J. Food Microbiol., 2016, 233, 44-51.

18 Z. Wang, T. Yue, Y. Yuan, R. Cai, C. Guo, X. Wang and C. Niu, J. Food Sci., 2012, 77, M643-M649.

19 J. Cui, K. Zhang, Q. Huang, Y. Yu and X. Peng, Anal. Chim. Acta, 2011, 688, 84-89.

20 M. Casanova, M. L. Gil, L. Cardeñoso, J. P. Martinez and R. Sentandreu, Infect. Immun., 1989, 57, 262-271.

21 J. Turkevich, P. C. Stevenson and J. Hillier, Discuss. Faraday Soc., 1951, 11, 55-75. 
22 Z. Wang, T. Yue, Y. Yuan, R. Cai, C. Guo, X. Wang and C. Niu, J. Food Sci., 2012, 77(11), M643-M649.

23 A. Beaussart, D. Alsteens, S. El-Kirat-Chatel, P. N. Lipke, S. Kucharíková, P. Van Dijck and Y. F. Dufrêne, ACS Nano, 2012, 6(12), 10950-10964.

24 W. Eck, G. Craig, A. Sigdel, G. Ritter, L. J. Old, L. Tang, M. F. Brennan, P. J. Allen and M. D. Mason, ACS Nano, 2008, 2, 2263-2272.

25 J.-Y. Byun, Y.-B. Shin, D.-M. Kim and M.-G. Kim, Analyst, 2013, 138, 1538-1543.

26 H. Hoffmann, S. Baldofski, K. Hoffmann, S. Flemig, C. P. Silva, V. I. Esteves, F. Emmerling, U. Panne and R. J. Schneider, Talanta, 2016, 158, 198-207.
27 Z. Wang, R. C. Beier, Y. Sheng, S. Zhang, W. Jiang, Z. Wang, J. Wang and J. Shen, Anal. Bioanal. Chem., 2013, 405, 40274037.

28 Y.-Y. Hsieh, P.-H. Hung and J.-Y. Leu, Molecular Cell, 2013, 50, 82-92.

29 H. J. Kim, H. R. Lee, C. S. Kim, Y. S. Jin and J. H. Seo, Enzyme Microb. Technol., 2013, 53, 174-180.

30 H. Rawsthorne and T. G. Phister, Int. J. Food Microbiol., 2006, 112, 1-7.

31 H. Wang, Z. Hu, F. Long, C. Guo, Y. Yuan and T. Yue, J. Food Prot., 2015, 78, 2052-2063. 